\title{
La géométrie et la vie des formes
}

\section{Geometry and the life of forms}

\section{Ruth Scheps ${ }^{1}$}

${ }^{1}$ docteur en génétique moléculaire (The Weizmann Institute of Science, Rehovot, Israël) ; productrice à France Culture et journaliste à la Radio Suisse Romande jusqu'en 2009 ; rédactrice en chef de la revue Mikhtav Hadash / La Nouvelle Lettre jusqu'en 2019. rscheps@hotmail.com.

RÉSUMÉ. Envisagée globalement, la vie des formes montre une même tendance à la complexification pour les formes naturelles et pour les formes géométriques résultant des mathématiques ou de l'art, avec une nette accélération pour ces dernières à l'époque moderne. Depuis le début du $X X^{\text {ème }}$ siècle, les avancées géométriques (géométries non-euclidiennes, théorie des catastrophes, géométrie algorithmique, théorie fractale) ont inspiré les artistes, en particulier ceux de l'abstraction géométrique à travers tous ses avatars - du suprématisme jusqu'à l'art numérique, en passant par l'art optique, l'art cinétique, l'art conceptuel et le minimalisme.

Cet article aborde la « vie des formes » de façons multiples : statuts respectifs de la forme et de la formation dans la nature, les arts et les sciences ; rôle du temps et du mouvement dans la perception des formes ; difficulté de l'idée de forme, aux échelles dimensionnelles extrêmes ; pertinence des notions de bord et de contenu en tant que critères déterminants des formes mathématiques ou artistiques ; limites de l'approche géométrique pour la connaissance des formes. On avancera enfin l'hypothèse d'une vie des formes excédant la géométrie, et exigeant une approche sensorielle ou spirituelle.

ABSTRACT. Viewed globally, the life of forms shows the same tendency to complexification for natural forms and for geometric forms resulting from mathematics or art, with a clear acceleration for the latter in modern times. Since the beginning of the 20th century, geometric advances (non-Euclidean geometries, catastrophe theory, algorithmic geometry, fractal theory) have inspired artists, particularly those of geometric abstraction through all its avatars - from suprematism to digital art, via optical art, kinetic art, conceptual art and minimalism.

This article addresses the "life of forms" in multiple ways: the respective status of form and formation in nature, the arts and the sciences; the role of time and movement in the perception of forms; the difficulty of the idea of form, at extreme dimensional scales; the relevance of the notions of edge and content as determining criteria of mathematical or artistic forms; the limits of the geometric approach to the knowledge of forms. Finally, the hypothesis will be put forward of a life of forms that goes beyond geometry and requires a sensory or spiritual approach.

MOTS-CLÉS. Géométrie, forme, morphogenèse, mathématiques, fractalité, numérique, abstraction géométrique, univers.

KEYWORDS. Geometry, form, morphogenesis, mathematics, fractality, digital, geometrical abstraction, universe.

\section{Introduction}

Tenter de saisir la vie des formes dans sa plus grande généralité, c'est la considérer dans le temps long : à l'échelle des temps géologiques pour les formes naturelles, à celle des temps historiques pour les formes géométriques résultant de l'art ou des mathématiques. Dans les deux cas, c'est une même tendance à la complexification qui s'observe, avec une nette accélération à partir du $\mathrm{XX}^{\text {ème }}$ siècle pour l'art et les mathématiques.

Cet article mettra à l'honneur l'histoire récente des interactions entre la géométrie et les arts plastiques. Nous évoquerons les avancées géométriques du $\mathrm{XX}^{\mathrm{ème}}$ siècle - géométries noneuclidiennes, théorie des catastrophes, géométrie numérique/algorithmique, théorie fractale.... Nous montrerons comment les artistes les ont intégrées et s'en sont inspirés, notamment à travers les divers courants de l'abstraction géométrique - du suprématisme à l'art numérique, en passant par l'art optique, l'art cinétique, l'art conceptuel et le minimalisme.

Notre approche de la « vie des formes » sera multiple. Nous évoquerons les statuts respectifs de la forme et de la formation, en nous appuyant sur certains artistes, savants et philosophes ; nous 
considérerons le rôle du temps et du mouvement dans la perception des formes ; nous accorderons une place à la théorie des catastrophes, qui a modélisé les changements de forme abrupts. Nous questionnerons également l'existence de formes aux échelles dimensionnelles extrêmes : sont-elles pertinentes pour caractériser les particules quantiques et l'univers dans sa globalité ?

Enfin nous examinerons la pertinence des notions de bord et de contenu en tant que critères déterminants des formes mathématiques ou artistiques, et nous ferons l'hypothèse d'une vie des formes excédant la géométrie, et exigeant une approche sensorielle ou spirituelle.

\section{Prendre forme}

La vie des formes (géométriques ou non) dépasse de loin leur assignation à la science géométrique. Le mot «forme » apparaît en moyen français au $\mathrm{XI}^{\text {ème }}$ siècle. Formé (!) à partir du latin forma, il désigne " l'ensemble des caractéristiques extérieures de quelque chose ${ }^{1}$-un être, un objet, un événement, une idée - ou bien leur façon de se matérialiser ou de se présenter. De manière plus scientifique, «forme» peut être défini comme toute l'information géométrique qui reste d'un objet lorsque l'emplacement, l'échelle et les effets de rotation sont filtrés. Dans tous les cas de figure, une forme reconnue comme telle, est indissociable de sa perception : même sa définition algébrique ne saurait la faire exister vraiment, à moins qu'elle soit complétée par le dessin géométrique.

\section{Premières géométries}

La connaissance des formes géométriques est encore bien plus ancienne que le mot qui les désigne. De fait, les humains ont toujours cherché à comprendre et à reproduire les formes qu'ils voyaient autour d'eux. Les premières formes géométriques dessinées apparaissent au Paléolithique, les premières notions de géométrie reconnues, vers 3000 av. J.-C., en Égypte, en Inde ancienne et chez les Babyloniens. Vers le $\mathrm{I}^{\mathrm{er}}$ ou le $\mathrm{II}^{\mathrm{e}}$ siècle av. J.-C., paraissent Les Neuf Chapitres sur l'art mathématique, texte fondamental des connaissances de la Chine ancienne, avec des calculs d'aires et de volumes, et une formulation du théorème de Pythagore. C'est la Grèce antique (dès -600), qui fera de la géométrie une science à part entière, en généralisant et établissant des lois à partir de nombreuses règles empiriques connues depuis longtemps. Les pythagoriciens (VI-V ${ }^{\text {ème }}$ siècles av. J.-C.) avaient déjà une connaissance empirique de trois solides - le tétraèdre (pyramide), l'hexaèdre (cube), le dodécaèdre. À leur suite, Platon a nommé cinq polyèdres réguliers, appelés depuis « solides de Platon »: le tétraèdre, l'hexaèdre, l'octaèdre, le dodécaèdre et l'icosaèdre ${ }^{2}$. Loin d'être purement mathématiques, ces figures de base s'inscrivent pleinement dans la philosophie platonicienne : dans La République, elles apparaissent comme des formes sensibles ne devant leur réalité qu'à leur participation aux essences intelligibles et transcendantes (elles-mêmes subordonnées au Bien suprême, simple et inconditionné) : "Les mathématiciens construisent leurs raisonnements, sans avoir à l'esprit ces figures elles-mêmes [les cinq solides de base] mais les figures parfaites dont elles sont les images visibles et que nul ne peut contempler autrement que par la pensée ${ }^{3}$. 》 Dans le Timée ${ }^{4}$, Platon présente les quatre éléments comme étant constitués de

\footnotetext{
${ }^{1}$ TLFi, Le Trésor de la langue française informatisé, 1971-1994. Parmi les substantifs directement dérivés, notons : formation, conformation, déformation, format, formateur, formatage, formalisme, formalisation, formule, formulaire, formalité, malformation, méforme, néoformation, plateforme - une richesse sémantique en accord avec l'ancienneté du terme et son caractère peu spécifique.

${ }^{2}$ Les cinq solides (polyèdres) de Platon et leurs faces (polygones) : tétraèdre ou pyramide (4 triangles équilatéraux) ; hexaèdre ou cube (6 carrés) ; octaèdre ( 8 triangles équilatéraux) ; dodécaèdre (12 pentagones réguliers), icosaèdre (20 triangles équilatéraux).
}

${ }^{3}$ Platon, La République, VI, §10. 
particules respectivement tétraédriques (Feu), cubiques (Terre), octaédriques (Air) et icosaédriques (Eau), la sphère du monde (le Tout) étant un dodécaèdre. Quelque soixante ans plus tard, Euclide offrait, avec ses Éléments, une étude mathématique complète des solides de Platon ${ }^{5}$ qui, en raison de leur esthétique et de leurs symétries, ont continué à être étudiés bien après les mathématiciens grecs.

\section{Géométriser la complexité}

Vers le début du $\mathrm{XX}^{\mathrm{ème}}$ siècle, la naissance de la géométrie non-euclidienne (Bernhard Riemann, 1854), de la théorie des quantas (Max Planck, 1900) et de la relativité (Albert Einstein, 1905) résulte en une conception spatio-temporelle du monde et produit des formes que les époques précédentes n'auraient pu imaginer : surface de Möbius (popularisée par l'œuvre de M. C. Escher), bouteille de Klein, projections de formes à plus de trois dimensions...). Qu'elles se réfèrent à des réalités physiques possibles ou impossibles, elles vont au-delà des formes idéales de la géométrie euclidienne, destinée à décrire les formes réelles de manière plus ou moins approchée.

Ces nouvelles théories et hypothèses mathématiques vont raviver l'intérêt des artistes pour les sciences (une tendance datant de l'invention de la perspective) et inspirer les démarches cubistes, constructivistes et surréalistes, "provoquant dans les références de l'art un dépaysement soudain, comme une abstraction avant l'abstraction $»^{6}$. Pour ne donner qu'un exemple de la façon dont les artistes se sont emparés de ces nouvelles constructions scientifiques, citons René Magritte, qui a su en jouer pour proposer des représentations paradoxales d'objets impossibles ${ }^{7}$, où les principaux effets de la profondeur sont contredits.

En tant que surfaces, volumes ou hypervolumes, les formes « vivent » au sein d'espaces linéaires ou courbes avec lesquels elles interagissent. Mais le sens d'une forme singulière tient aussi aux autres formes auxquelles elle peut être associée. Pour le mathématicien, «ce qui est intéressant, ce que dit véritablement une forme, c'est une famille à laquelle elle est agrégée, un groupe dans lequel elle se range. Et pour voir, comme pour dire, dans quelle famille une forme est rangée, $[\ldots]$ une des solutions consiste à déployer la forme pour lui faire engendrer une famille de formes, dont elle sera en quelque sorte le représentant. Et un bon moyen, c'est de la déployer dans le temps, de faire se mouvoir la forme ${ }^{8}$. » Enfin, une forme géométrique peut être engendrée à partir d'une équation algébrique faisant intervenir les nombres imaginaires. Il est à noter que dans ce cas, la forme

\footnotetext{
${ }^{4}$ Timée : un des derniers dialogues de Platon, écrit vers 360 av. J.-C. Le philosophe pythagoricien Timée de Locres réfléchit sur l'origine et la nature du monde physique et de l'âme humaine, tout en abordant les questions liées à la connaissance scientifique et au rôle des mathématiques dans l'explication du monde.

${ }^{5}$ Un polyèdre régulier est un solide de Platon, si et seulement si, $1:$ toutes ses faces sont des polygones réguliers convexes isométriques, c'est-à-dire superposables ; 2 : aucune de ses faces ne se coupe, excepté sur les arêtes ; 3 : le même nombre de faces se rencontre à chacun de ses sommets. De plus, en géométrie euclidienne, la somme des angles au sommet des polygones réguliers doit être strictement inférieure à $360^{\circ}$.
}

\footnotetext{
${ }^{6}$ Jean de Loisy, « Formes mathématiques », cat.exp. Formes simples, dir. Jean de Loisy ; exposition au Centre Pompidou-Metz, 13 juin - 5 novembre 2014, éditions du Centre Pompidou-Metz / H Fondation d'entreprise Hermès, p. 140.
}

\footnotetext{
${ }^{7}$ Objet impossible : représentation d'objet fictive, contraire aux lois physiques connues. Le triangle de Penrose (également exploité par M. C. Escher) en est un exemple emblématique : le dessin censé être l'exacte représentation, en projection sur un plan, d'un objet réel tridimensionnel, est ambigu car on ne peut l'interpréter comme tel. Par conséquent, l'objet théorique, qui devrait correspondre au dessin, est impossible. D’une manière générale, ces dessins appliquent les règles de la perspective de manière " perverse ", en plaçant des indices contradictoires sur la position et la taille des objets.
}

\footnotetext{
${ }^{8}$ Jean Dhombres, "Qu'est-ce qu'une forme ? ", Les sciences de la forme aujourd'hui, collectif, entretiens avec Émile Noël, éditions du Seuil, " Points Sciences », 1994, p. 18.
} 
géométrique obtenue est une interprétation particulière de l'équation de départ, laquelle en admet plusieurs.

En topologie (étude des formes géométriques et de leurs relations), peu de mathématiciens auront autant contribué à la vie des formes que René Thom ${ }^{9}$. Le bel article que lui consacre l'Encyclopadia Universalis, intitulé «De l'algèbre à la géométrie et de la géométrie au monde », souligne que «René Thom est un des maîtres incontestés de la géométrie. Comme celle de Riemann, comme celle de Poincaré (dont il partage la perspective intuitionniste, synthétique, holiste et l'aversion corrélative pour le formalisme pur), son œuvre mathématique est dominée par une interrogation essentielle : le "retour" de l'algébrique vers le géométrique. » La théorie des catastrophes met en valeur une approche qualitative de la géométrie, appelée à devenir une science phénoménologique. Les « catastrophes » de Thom (dont il se plaisait à dire qu'elles n'ont jamais tué personne !) sont des changements de forme soudains, des discontinuités décrites sous la forme de surfaces mathématiques abstraites. Thom a défini sept catastrophes élémentaires, sept formes pouvant être contrôlées avec quatre paramètres au maximum - les quatre dimensions de notre environnement : longueur, largeur, hauteur et temps. En évoluant, ces sept formes aboutissent à des singularités, autrement dit, à des points de discontinuité : le pli, la queue d'aronde, la fronce, le papillon, l'ombilic elliptique (l'extrémité d'une aiguille), l'ombilic parabolique (le champignon), l'ombilic hyperbolique (la crête d'une vague déferlante). Cette théorie a suscité un immense engouement chez de nombreux scientifiques et chez des artistes comme Salvador Dali, auteur d'une toile nommée Queue d'aronde (1983). Cependant, les tentatives visant à l'appliquer aux sciences humaines, notamment en linguistique et en psychologie, ont été vigoureusement contestées.

Dans les années 1970, grâce à la conception assistée par ordinateur (CAO), la géométrie s'est enrichie d'une nouvelle discipline, la géométrie algorithmique. L'outil informatique permettait désormais aux dessinateurs de modéliser des surfaces en trois dimensions (3D), ce qui ouvrait de nouveaux champs d'application aux artistes. Des formes tridimensionnelles inaccessibles à l'œil humain sont devenues mesurables, de l'échelle atomique à l'échelle astronomique. Mais il s'agissait encore de formes relativement simples. C'est l'apparition de données 3D massives et structurées par la géométrie algorithmique, qui permettra de représenter des formes complexes. Une question importante à cet égard concernait la représentation de formes géométriques continues par des modèles discontinus ${ }^{10}$, ce qui nécessitait de pouvoir caractériser la complexité d'un objet géométrique. Ce sera fait par Herbert Federer (fondateur de la théorie de la mesure géométrique) à l'aide d'une quantité, baptisée la « portée » (reach en anglais), qui résume à elle seule la façon dont l'objet est géométriquement plongé dans l'espace. Ce concept, qui tient compte à la fois de la courbure de l'objet (une notion locale) et de son étranglement (une notion globale), a été appliqué notamment aux structures à très grande échelle de l'univers (galaxies et amas de galaxies).

\footnotetext{
${ }^{9}$ René Thom (1923-2002) : mathématicien français, auteur de la théorie des catastrophes dans les années 1960. Celle-ci a été popularisée dans les années 1970 par le mathématicien Christopher Zeeman, qui a continué à l'appliquer aux sciences biologiques et comportementales. René Thom a reçu la médaille Fields (l'équivalent mathématique du prix Nobel) en 1958 pour ses travaux en topologie.

${ }^{10}$ Modèles discontinus (ou discrets) : deux exemples emblématiques sont : $1^{\circ}$ les diagrammes de Voronoï, qui représentent les relations de proximité et permettent de modéliser des phénomènes de croissance en toutes dimensions; $2^{\circ}$ le modèle des triangulations, qui est au cœur de l'interaction entre géométrie et calcul.
} 


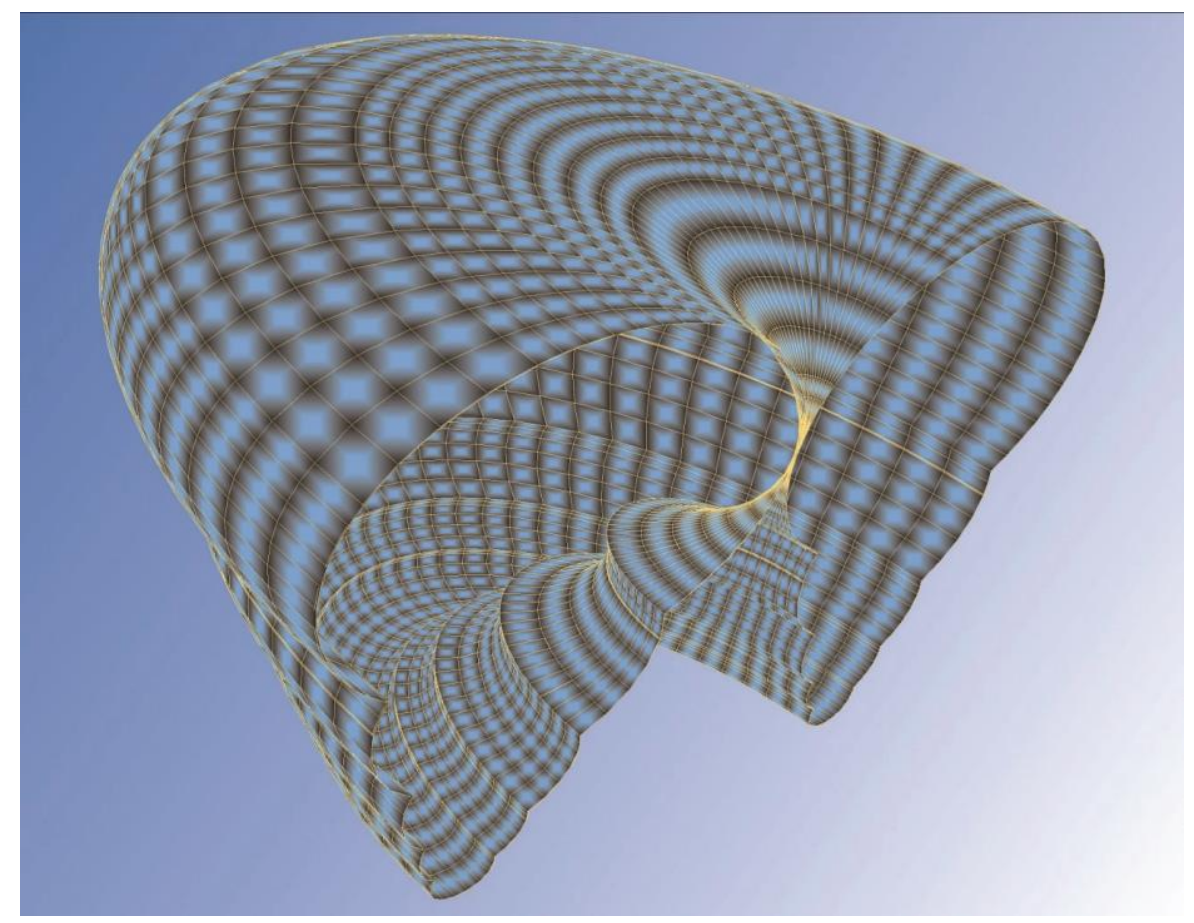

Figure 1. Reuven Berman Kadim, Hovering Object \#1. Digital image, 1997. "Artist's Estate, Reuven Berman Kadim, "Geometric Art, The Hidden Order of Nature", pub. Yedioth Books, Israel, 2010".

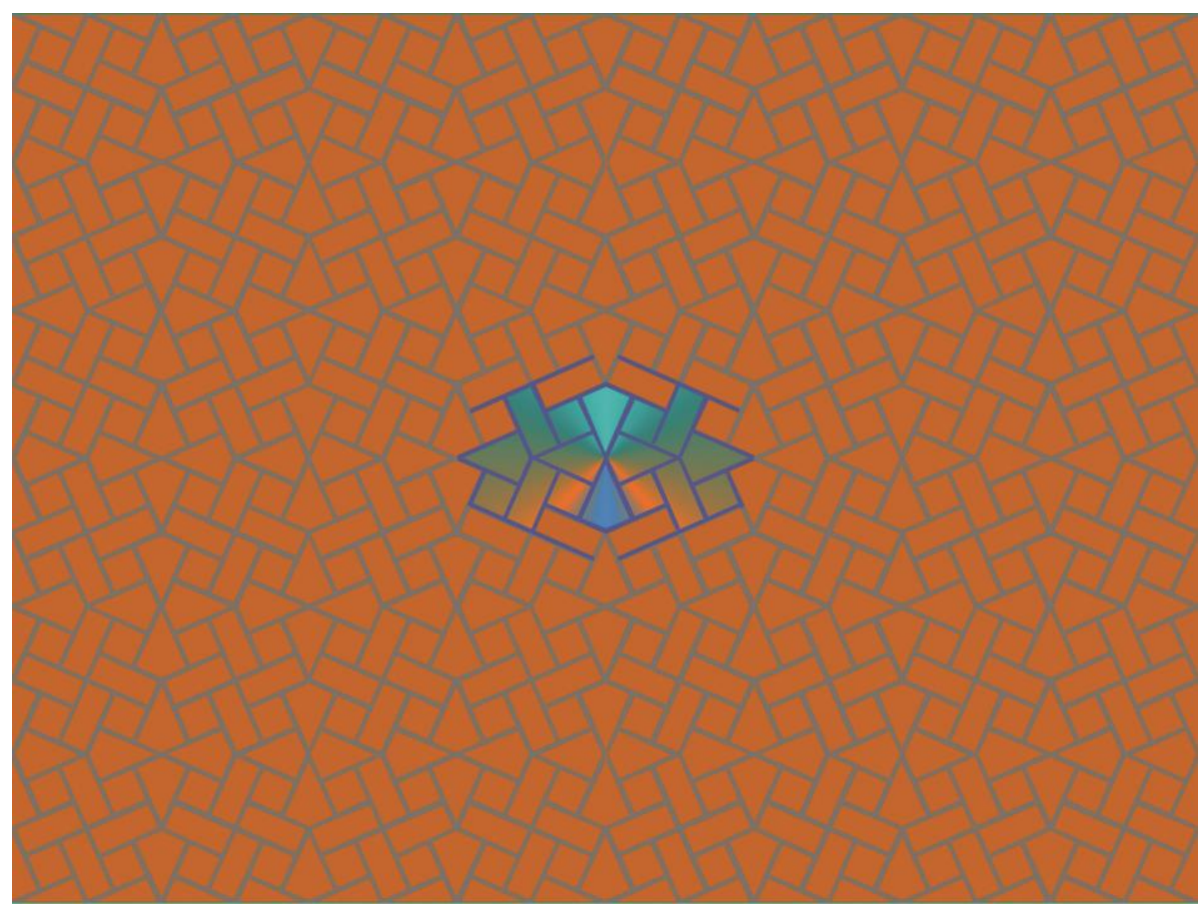

Figure 2. Reuven Berman Kadim, Paving B, Digital image, 1996. "Artist's Estate, Reuven Berman Kadim, "Geometric Art, The Hidden Order of Nature", pub. Yedioth Books, Israel, 2010".

\section{Morphogenèses}

Les formes identifiées comme telles, sont des constructions de l'esprit visant à réduire le caractère mouvant de la réalité. Le philosophe Henri Bergson remarque : «La vie est une évolution. Nous concentrons une période de cette évolution en une vue stable que nous appelons une forme, et, quand le changement est devenu assez considérable pour vaincre l'heureuse inertie de notre perception, nous disons que le corps a changé de forme. Mais, en réalité le corps change de forme à tout instant. Ou plutôt il n'y a pas de forme, puisque la forme est de l'immobile et que la réalité est mouvement. Ce qui est réel, c'est le changement continuel de la forme : la forme n'est qu'un 
instantané pris sur une transition ${ }^{11}$. » Cependant, en art comme en science, la saisie de ces instantanés (les formes) a été un préalable nécessaire à celle de leurs transitions (leur formation).

Dans la nature, certaines formes évoquent immédiatement le mouvement dont elles procèdent. C'est le cas des spirales, que l'on trouve à toutes les échelles de grandeur, depuis les galaxies jusqu'à l'ADN (doublement spiralé), en passant par des formes visibles à l'œil nu, comme celles de l'ammonite, de la pomme de pin, de la fleur de tournesol, etc.

Dans le monde animal, les morphogenèses sont autant constructives que destructives. À chaque étape de l'embryogenèse, il faut en effet compter avec l'apoptose, cette mort cellulaire spontanée, qui contribue de manière essentielle à façonner les organes et les membres ${ }^{12}$; dans le cas de la main, par exemple, c'est par une succession d'apoptoses que les doigts se libèrent progressivement de leur gangue en forme de moufle.

La complexité des formes naturelles défie souvent la géométrie. Avant l'apparition de la géométrie algorithmique, les artistes devaient donc emprunter d'autres voies pour exprimer leur essence. Avec son Homme de Vitruve (1490), Léonard de Vinci a montré que l'homme, représenté bras et jambes écartés, pouvait être inscrit dans les formes géométriques les plus simples et parfaites : le cercle et le carré. Plus près de nous, le sculpteur Jean Arp, confronté à la diversité des formes complexes, tend vers l'épure : «À Ascona, je dessinais au pinceau avec de l'encre de Chine des branches cassées, des racines, des herbes, des cailloux que le lac avait rejetés sur le rivage. Je simplifiais et unifiais ces formes en ovales mouvants, symboles de l'éternelle métamorphose et du devenir des $\operatorname{corps}^{13}$. » Pourrait-on aller plus loin dans la compréhension du devenir forme, par exemple en dévoilant ses ressorts secrets ? L'historien de l'art Henri Focillon le suggère : «Rien n'est plus tentant - et rien, dans certains cas, n'est mieux fondé - que de montrer les formes soumises à une logique interne qui les organise. De même que, sous l'archet, le sable répandu sur une plaque vibrante se meut pour dessiner diverses figures qui s'accordent avec symétrie, de même un principe caché, plus fort et plus rigoureux que toute fantaisie inventive, appelle l'une à l'autre des formes qui s'engendrent par scissiparité, par déplacement de tonique, par correspondance ${ }^{14}$. »

Ainsi, qu'elles soient naturelles, artistiques ou scientifiques, les formes informent aussi : sur la «logique interne qui les organise » comme sur les objets, les êtres ou les symboles auxquels elles renvoient.

\section{Art et géométrie}

Les arts influent sur le monde, notamment à travers des œuvres qui en modifient la perception. La modernité picturale a été particulièrement active à cet égard. En témoignent les divers courants qui ont surgi au $\mathrm{XX}^{\text {ème }}$ siècle - du cubisme à toutes les formes d'art numérique - comme pour inquiéter nos certitudes quant à la nature de la réalité. Bien des distinctions scientifiques ou philosophiques traditionnelles en ont été bousculées : entre l'espace et le temps, le réel et le virtuel, le réel et l'imaginaire, le vrai et l'illusoire. Le champ des possibles s'étant démultiplié, l'œuvre participe désormais à son propre devenir.

\footnotetext{
${ }^{11}$ Henri Bergson, L'Évolution créatrice, 1907, Paris, PUF, 1959, p. 301-302.

12 Jean-Claude Ameisen, La sculpture du vivant. Le suicide cellulaire ou la mort créatrice, éditions du Seuil, « Points sciences humaines », 2003.

${ }^{13}$ Jean Arp, « Jalons », Jours effeuillés, Poèmes essais, souvenirs, Paris, Gallimard, 1966, à la note 2, p. 357.

${ }^{14}$ Henri Focillon, Vie des formes, Paris, PUF, 1934, $8^{\text {ème }}$ édition, 2004, p. 12.
} 


\section{L’art géométrique avant la lettre}

D’une certaine façon, l'art géométrique a toujours existé : des signes géométriques préhistoriques ont été trouvés dans presque tous les sites d'art pariétal d'Europe occidentale - mais leur signification demeure inaccessible ; dans l'Antiquité grecque, les polygones, construits à la règle et au compas, ont été utilisés en architecture ; le Haut Moyen Âge a vu naître l'art des entrelacs, qui s'est développé en Europe comme en Orient où il a donné lieu aux arabesques. Mais c'est l'invention de la perspective, au $\mathrm{XV}^{\text {ème }}$ siècle, qui a été la plus décisive pour l'art géométrique : ses diverses techniques ont permis aux artistes de représenter la profondeur d'un objet ou d'une scène sur une surface plane, et elle a fasciné aussi bien Léonard de Vinci que les peintres modernes liés aux diverses déclinaisons de l'art géométrique.

Au début du XX $X^{\text {ème }}$ siècle, la naissance du cubisme (Picasso et Braque, 1900) a été une étape déterminante dans l'histoire de l'art. Témoin privilégié de cette peinture, Guillaume Apollinaire en a souligné la pureté scientifique : «Un Picasso étudie un objet comme un chirurgien dissèque un cadavre. [...] L'aspect géométrique qui a frappé si vivement ceux qui ont vu les premières toiles scientifiques venait de ce que la réalité essentielle y était rendue avec une grande pureté et que l'accident visuel et anecdotique en avait été éliminé ${ }^{15}$. » Il ne s'agissait plus de représenter la réalité, mais d'en fragmenter les formes, puis de les recomposer dans diverses configurations géométriques abstraites, propres à montrer des objets de plusieurs points de vue à la fois. Cet exercice du regard fut aussi un exercice pour l'esprit, confronté à la coexistence de l'un et du multiple, du bidimensionnel et du tridimensionnel. Sans point de vue privilégié, l'objet cubiste allait faire du spectateur traditionnel un regardeur actif, capable de voir/concevoir, derrière l'unité brisée d'un objet peint, une multiplicité d'objets réels.

\section{De l'abstraction géométrique à l'art numérique}

L'abstraction géométrique proprement dite, qui s'élabore au sortir de la première Guerre mondiale, peut être considérée comme une seconde formulation de l'abstraction, d'abord spiritualiste et d'expression plutôt lyrique, fondée essentiellement par Vassily Kandinsky (19101913). Cet art géométrique abstrait se développera entre les deux guerres, par exemple sous les appellations d'art construit ${ }^{16}$ et d'art concret $^{17}$.

Apparu en même temps que le constructivisme russe, le suprématisme, théorisé par Kasimir Malevitch en $1915^{18}$, parachève la révolution du regard qu'avait entamée le cubisme : délestées de toute référence réaliste ou symbolique, ses compositions de formes pures ne renvoient qu'à ellesmêmes. Après Carré noir sur fond blanc (1913), l'artiste poursuit sa quête mystique d'un monde vide de représentation, et aboutit à Carré blanc sur fond blanc (1918). Sommet ou impasse de la peinture ? En tout état de cause, l'abstraction géométrique avait atteint une limite qui appelait à son

\footnotetext{
${ }^{15}$ Guillaume Apollinaire, "Sur la peinture », II et VII, dans (Méditations esthétiques) Les Peintres cubistes, Paris, Figuière, 1913.

${ }^{16}$ Art construit : abstraction géométrique qui intègre le hasard, tout en restant dans la rigueur de la composition picturale. Elle se caractérise à la fois par sa logique et par sa grande diversité.

${ }^{17}$ Art concret : notion proposée en 1930 par Theo van Doesbourg, fondateur du groupe Art concret, et rédacteur de la revue De Stijl. « Peinture concrète et non abstraite, parce que rien n'est plus concret, plus réel qu'une ligne, qu'une couleur, qu'une surface » (Manifeste de l'art concret, cité par Michel Seuphor dans L'Art abstrait, vol. 1, p. 10). L'art concret se veut plus rigoureux que l'abstraction traditionnelle, en substituant à la subjectivité de l'artiste, l'application de systèmes et de programmes prédéterminés et objectivement contrôlables. Ce mouvement se poursuivra après la seconde Guerre mondiale, jusque vers 1960 .
}

${ }^{18}$ Kasimir Malevitch, Manifeste suprématiste : Le Nouveau Réalisme pictural, 1915. 
renouvellement. Celui-ci sera progressif : le géométrisme abstrait « réaliste » de Piet Mondrian (dès 1917) ne cherche pas à exprimer la réalité de la nature, mais son essence. Avec une remarquable économie de moyens, Mondrian se détourne peu à peu des formes et des couleurs naturelles, délaissant en particulier la courbe et la couleur verte pour privilégier la droite et les trois couleurs primaires - rouge, jaune et bleu -, le blanc lui servant de fond et le noir, de délimitation entre les couleurs. Il nommera «néo-plastique » ce système de représentation abstraite auquel l'a conduit son cheminement intérieur et par lequel il entend matérialiser l'Absolu. Pour le philosophe et historien de l'art Hubert Damisch (1958), « un tableau de Mondrian contredit au monde qui est le nôtre, et en suggère un autre. [Il lui est assigné] une fonction précise : celle d'imprimer dans la mémoire visuelle un schème d'organisation de l'espace qui fonctionnerait ensuite comme une grille, laquelle n'attendrait plus que d'être reportée sur le monde pour l'informer à neuf ${ }^{19}$. »

L'abstraction géométrique avait abouti à une radicalité extrême qui en menaçait le développement. Le défi était désormais de la prolonger, mais sans la renier. Il sera relevé, entre 1950 et 1970, par l'art optique (Op Art aux États-Unis) et l'art cinétique ${ }^{20}$, qui prennent en compte les connaissances scientifiques pour mettre en avant le rapport entre l'art et la perception optique une démarche initiée par Victor Vasarely, considéré depuis comme le père de l'art optique. En créant des illusions optiques, c'est la perception du mouvement que Vasarely cherche à approfondir : par leur mise en perspective, les formes géométriques simples et répétées de ses tableaux, incitent le regard à se déplacer de l'une à l'autre et produisent une impression spectaculaire de volume, concave ou convexe.

Les formes géométriques simples n'ont pas encore dit leur dernier mot : poussant la simplicité à l'extrême, le minimalisme qui surgit au début des années 60, hérite directement de Malevitch dans sa quête de l'Absolu, et s'oppose à l'expressionisme abstrait des années 50. Barnett Newman, l'un de ses artistes les plus importants, va parfois même jusqu'à ignorer la forme au profit de la seule couleur.

En 1961, certains artistes optiques-cinétiques fondent un collectif, le GRAV ${ }^{21}$, dont un représentant emblématique est François Morellet, figure majeure du minimalisme. Composées de formes géométriques simples, ses œuvres sont strictement planifiées et comportent souvent une trame systématique, contrebalancée par l'intervention ludique du hasard. Il y introduit également des sources de lumière (ampoule, néon) qui créent des phénomènes d'interférence et de clignotement, destinés à déstabiliser la perception.

Autre artiste phare de l'art minimal, Sol LeWitt, qui multiplie les combinaisons de lignes dans un format carré, est également identifié à l'art conceptuel ${ }^{22}$, aboutissement logique du minimalisme. Dans une œuvre conceptuelle, ce ne sont pas les propriétés formelles esthétiques qui priment, mais

\footnotetext{
${ }^{19}$ Hubert Damisch, cité par Éric de Chassey, L'abstraction avec ou sans raisons, Gallimard, coll. « Art et artistes », 2017, p. 29.

${ }^{20}$ Art cinétique : révélé au public en 1955 par l'exposition Le Mouvement, proposée par Vasarely à la galerie Denise René ; avec des œuvres de Marcel Duchamp, Alexander Calder, Jesús Rafael Soto, Yaacov Agam, Jean Tinguely et Pol Bury, etc. Art optique : présenté pour la première fois en 1965, lors de l'exposition The Responsive Eye, au MoMa de New York ; avec des œuvres de 99 artistes dont Josef Albers, un des initiateurs de l'art optique, Victor Vasarely, son père officiel, et de nombreux artistes de l'art cinétique en Europe.
}

${ }^{21}$ GRAV : Groupe de Recherches d'Art Visuel, créé par François Morellet avec cinq autres artistes : Francisco Sobrino, Horacio Garcia Rossi, Julio Le Parc, Yvaral et Joël Stein. Son objectif est la création d'un art fondé sur le mouvement, la non-subjectivité de l'artiste et la participation du public.

${ }^{22}$ Art conceptuel : cf. «Quand les attitudes deviennent forme », exposition fondamentale organisée par Harald Szeemann entre le 22 mars et le 27 avril 1969 à la Kunsthalle de Berne et réunissant de nombreux artistes conceptuels. 
le concept ou l'idée de l'art qu'elle incarne : «Lorsqu'un artiste recourt à une méthode modulaire multiple, il choisit habituellement une forme simple et disponible. La forme, elle-même, a une importance très réduite : elle devient la grammaire de l'œuvre dans son entité. [...] L'arrangement devient la fin et la forme devient le moyen ${ }^{23}$. » Ce qui en résulte n'est rien moins qu'une nouvelle forme de perception de l'œuvre.

Dans les années 50, l'apparition des premiers ordinateurs révolutionne l'art géométrique : de nouvelles formes géométriques sont construites, générant encore de nouvelles manières de voir et d'analyser la peinture. Venue du minimalisme (avec un vocabulaire formel fondé sur la ligne, le cercle, le carré ou le méandre), l'artiste hongroise Véra Molnár (née en 1924) voit dans l'ordinateur utilisé à des fins créatrices, un outil libérateur par rapport à l'héritage classique qu'elle juge sclérosé, et à partir de 1968, elle devient l'une des pionnières de cet art numérique - ou art algorithmique aux possibilités apparemment infinies. Un art multiforme ${ }^{24}$, qui séduit de plus en plus de jeunes artistes, telle Sarah Morris (née en 1967) chez qui « les structures architecturales, devenues des structures picturales minimales, sont à la fois des déconstructions et des reconstructions plastiques qui mêlent les codes de la $2 \mathrm{D}$ à ceux de la $3 \mathrm{D}$, des représentations planes et des volumes. Ces derniers peuvent se présenter séparément au sein de certaines toiles, ou imbriqués dans d'autres. Les espaces représentés sont aussi d'autant plus complexes qu'ils jouent d'étirements, d'aplatissements et de perspectives d'angles ${ }^{25}$. »

Le milieu des années 80 voit émerger un nouveau courant de l'art numérique, l'art fractal nommé en référence à une découverte du mathématicien Benoît Mandelbrot ${ }^{26}$, la théorie des fractales - compositions qui répètent à l'infini le même motif géométrique, à différentes échelles (autosimilarité). Ces structures, qui existent aussi dans la nature, ont donné lieu à la fractalité, une nouvelle vision du monde largement inspirée par la notion d'échelle et les rapports entre le tout et le détail. Certains artistes s'empareront de cette vision fractale du monde pour la traduire dans leurs œuvres, qui incarnent la nouvelle fusion des mathématiques et de l'art.

En fin de compte, ce qu'aura incarné l'art géométrique, à travers tous ses avatars, c'est l'adieu à la subjectivité et au monde des objets : l'abstraction géométrique s'est tournée vers l'objectivité, la neutralité, l'anonymat et la vérité ; le suprématisme, vers l'infini ; l'art numérique, vers la sérialité et la perfection formelle.

\footnotetext{
${ }^{23}$ Sol LeWitt, "Paragraphs on Conceptual Art” in Artforum, juin 1967.

${ }^{24}$ Art numérique : s'est développé en tant que genre artistique depuis la fin des années 1950. II regroupe un ensemble varié de formes d'art utilisant des dispositifs numériques (ordinateur, interface ou réseau) - par exemple le net.art (œuvres interactives conçues par, pour et en lien avec le réseau Internet), l'art robotique ou la photographie numérique, avec des sous-catégories spécifiques telles que l'art audiovisuel, l'art génératif, l'art interactif. Les environnements de réalité virtuelle (RV) et de réalité augmentée (RA) en sont aujourd'hui des catégories importantes. Enfin, l'intégration de la vie artificielle et de l'intelligence artificielle aux créations numériques, en renouvelle les perspectives.
}

${ }^{25}$ Elsa Ayache, L'informatique, outil et médium du peintre, vers une pratique du "lâcher-prise ", thèse de doctorat, Université Michel de Montaigne - Bordeaux III, 2018.

\footnotetext{
${ }^{26}$ Benoît Mandelbrot (1924, Varsovie - 2010, Cambridge, États-Unis). Mathématicien franco-américain. Découvreur des fractales, une nouvelle classe d'objets mathématiques, qu'il présente en 1975 dans l'ouvrage de référence Les Objets fractals - Forme, hasard et dimension, trad., Flammarion, 1975. Cette découverte a ouvert un champ mathématique devenu célèbre - la géométrie fractale - et montré l'étendue de ses applications dans les domaines les plus variés (biologie, physique, finance, etc.).
} 


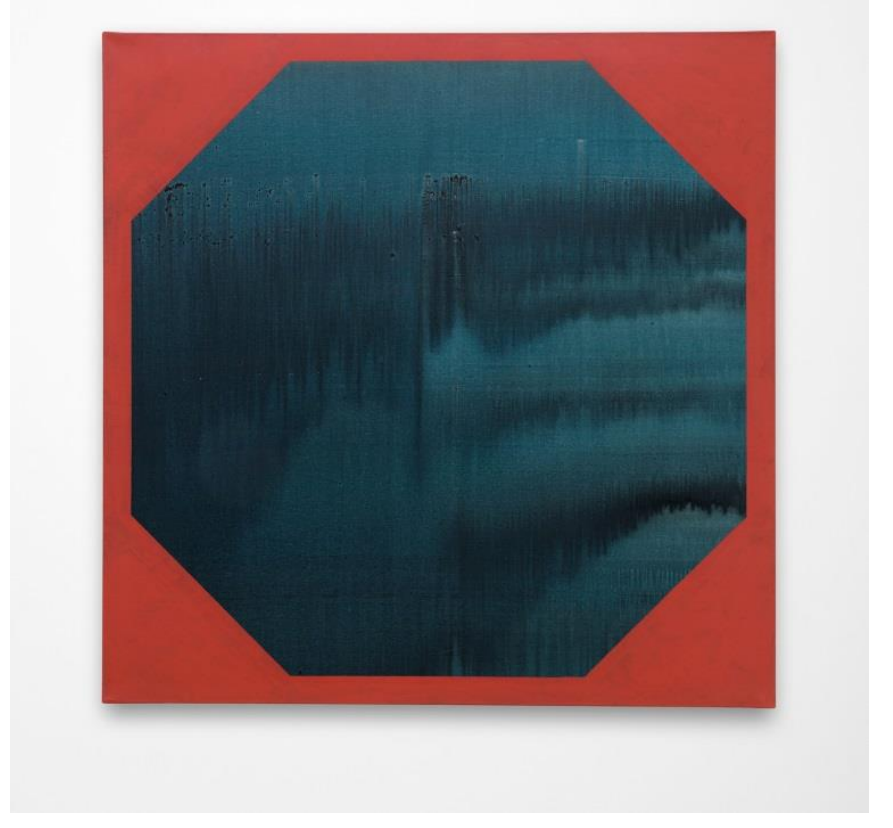

Figure 3. Emmanuel Van der Meulen, Quadrum, 2017, acrylique sur toile, $130 \times 130 \mathrm{~cm}$. Courtesy de l'artiste et Galerie Allen, Paris.

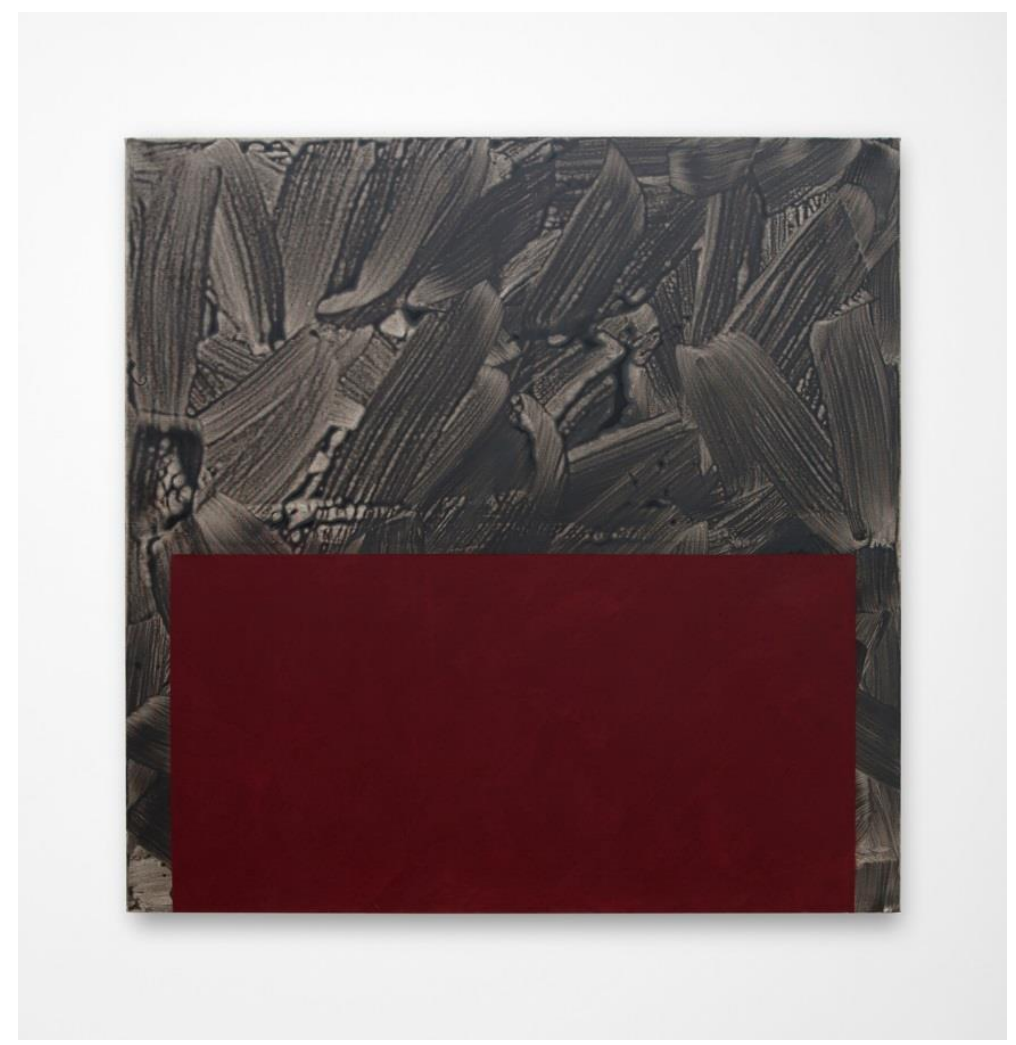

Figure 4. Emmanuel Van der Meulen, Bethel, 2017, acrylique sur toile, $130 \times 130 \mathrm{~cm}$. Courtesy de l'artiste et Galerie Allen, Paris. 


\section{Trois héritiers de l'art géométrique}

\section{Reuven Berman Kadim ${ }^{27}$}

Tout l'œuvre de Reuven Berman Kadim relève de l'art géométrique abstrait. Au sein de ce vaste courant artistique, sa trajectoire l'a conduit vers l'art numérique - en particulier l'art fractal. L'organisation géométrique de la matière et les motifs géométriques de l'art islamique, sont ses deux grandes sources d'inspiration. L'artiste considère que les modèles géométriques créés par la théorie fractale, reproduisent 1'ordre sous-jacent des phénomènes naturels dits « chaotiques ». Par ailleurs il repère le même principe de répétition formelle dans les motifs géométriques de l'art islamique et dans les systèmes fractals.

Comme l'art des motifs islamiques, dans lequel l'artiste voit une métaphore visuelle de la force créatrice universelle, les objets sont à ses yeux des métaphores de l'ordre plus complexe qui les fonde. L'œuvre éminemment poétique, Objet flottant \#1 (fig. 1), est issue de la suite de Fibonacci ${ }^{28}$. Créée à l'aide d'un programme d'ordinateur, elle est emblématique des motifs complexes que cet outil lui permet de développer, et il n'est pas certain qu'elle aurait pu être réalisée sans ce programme.

L'œuvre Pavage (fig. 2), dans la section «Rectangles et angles » du livre Geometric Art. The Hidden Order of Nature, se compose de quelques figures géométriques simples et parfaitement juxtaposées : carré, rectangle, cerf-volant. Dans la partie centrale de l'image, l'uniformité du pavage semble contredite par le jeu des couleurs, et l'œil est irrémédiablement attiré vers ce centre qui se détache nettement du reste de l'image. Comme si toute la partie périphérique n'était destinée qu'à valoriser sa figure complémentaire.

\section{Emmanuel Van der Meulen ${ }^{29}$}

Pour Emmanuel Van der Meulen, la géométrie n'est pas un but en soi mais un outil, que l'artiste utilise sans dissocier la forme de la couleur. Dans ses tableaux, qui comportent toujours une forme centrale et des éléments périphériques, chaque point de la toile a la même importance - un principe qui donne à l'œil «l'occasion d'aller chercher un peu partout » : ce qui apparaît dans la peinture dépend aussi de ce que le regard y trouve au cours de ses déambulations.

\footnotetext{
${ }^{27}$ Reuven Berman Kadim (1929, Philadelphia, USA - 2014, Rehovot, Israël). Identifié à l'art géométrique abstrait. Critique d'art pour The Jerusalem Post (1961-1972) et pour le journal Yedioth Aharonoth (1966-73). Commissaire en 1983 de la Biennale Internationale de Sao Paulo, Brésil. À partir de 1995, a travaillé exclusivement à l'aide de l'ordinateur. Bibliographie sélective :
}

Marc Scheps, Reuven Kadim [Berman] - Patterns : An East-West Symbiosis, Tel Aviv Museum of Art, 2004.

Reuven Berman Kadim, Geometric Art. The Hidden Order of Nature, Gil Goldfine editor, Tel Aviv, Yedioth Ahronoth/ Chemed Books, 2010.

\footnotetext{
${ }^{28}$ Suite de Fibonacci : en mathématiques, suite d'entiers dans laquelle chaque terme est la somme des deux termes qui le précèdent. Elle commence par les termes 0 et 1 . Cette suite est liée au nombre d'or, $\varphi$ (phi), qui intervient dans l'expression du terme général de la suite. Les quotients de deux termes consécutifs de la suite de Fibonacci sont les meilleures approximations du nombre d'or.
}

\footnotetext{
${ }^{29}$ Emmanuel Van der Meulen est né en 1972 à Paris. Diplômé de l'École Nationale Supérieure des Beaux-Arts de Paris en 2001, il a été pensionnaire de l'Académie de France à Rome / Villa Médicis en 2012-2013. Son travail est représenté par la Galerie Allen, Paris. Expositions récentes : Turns \#2 (Galerie Allen, Paris, 2020), Chronique d'une collection : Embarquez-vous ! (FRAC GrandLarge, Dunkerque, 2020), Opsis (Exposition personnelle, Galerie Allen, Paris, 2019), 26 x Bauhaus (Institut Français, Berlin, 2019), Everybody's looking for something (La Salle de Bain, Lyon, 2019), Paris-Peinture (Le Quadrilatère, Beauvais, 2018), Fables, Formes, Figures (MABA, Nogent-sur-Marne, 2018), Quod Apparet (Exposition personnelle, Galerie Allen, Paris, 2017).
} 
Quadrum (fig. 3) est un tableau carré - la forme géométrique adoptée par l'artiste. Ici, il s'agit du cadre définissant explicitement l'octogone irrégulier qui le remplit largement. Mais ce qui se joue entre le cadre et l'octogone inscrit, dépasse le jeu formel et relève tout autant de la complémentarité des couleurs et de l'opposition des techniques picturales : la rigueur lisse du cadre-fenêtre et les larges aplats de couleur inclus produisent une rupture visuelle qui invite au voyage intérieur.

Le tableau Bethel ${ }^{30}$ (fig. 4) illustre de manière frappante la «double négation » qui intéresse l'artiste. La partie inférieure est une forme géométrique simple, un rectangle allongé dont les petits côtés ne touchent pas les bords verticaux du tableau, qui semblent l'encadrer. La première négation vient de ce que son grand côté inférieur « a l'air de reposer sur la limite inférieure du tableau, ce qui est d'autant plus absurde qu'un tableau, a priori, est accroché au mur et non posé par terre ${ }^{31}$. $\gg$ La seconde négation vient de la partie supérieure, qui semble réfuter tout ce qu'affirme le rectangle inférieur (équivalent pictural de l'autel de pierre érigé par Abram en Genèse 12,7 pour souligner l'importance du lieu) : des traits amples et tumultueux viennent buter contre la compacité du rectangle-pierre dont le rouge profond recouvre partiellement les couleurs neutres du haut. Deux énergies contraires et puissantes comme l'ordre et le chaos, invitent ici notre regard à se déplacer, indéfiniment.

\section{Esther Stocker $^{32}$}

Digne héritière de l'art abstrait, géométrique et optique, Esther Stocker l'a enrichi de ses propres obsessions artistiques, scientifiques et logico-philosophiques.

Regarder les œuvres d'Esther Stocker (peintures, sculptures, installations), est une expérience aussi passionnante que déstabilisante. Généralement élaborées à partir d'une grille en noir et blanc, elles sont envahies de subtils accrocs, distorsions et décrochages, qui viennent perturber la régularité attendue. Par ses constructions atypiques, l'artiste joue avec la géométrie des formes, et avec nos propres capacités à faire varier notre regard. L'incertitude qui émane de ses tableaux et installations, tient autant à la dialectique de l'ordre et du désordre qu'à celles de la forme et du fond, du simple et du complexe, ou encore du précis et du flou, rendus visuellement et mentalement interchangeables.

Comme la plupart des œuvres d'Esther Stocker, la peinture Sans titre 2010 (fig. 5) est une grille en noir et blanc, composée ici de rectangles noirs délimités par des traits blancs. Mais d'autres lignes ou parties de grilles, diversement orientées et savamment superposées à la grille primaire, en ont détruit la régularité. Ne trouvant pas où s'accrocher, le regard erre d'un point à un autre, et l'esprit logique est mis en échec.

\footnotetext{
${ }^{30}$ Bethel (ou Beth-El, en hébreu « Maison de Dieu ») est une localité des hautes terres du pays de Canaan, qui se trouverait à $10 \mathrm{~km}$ au nord de Jérusalem, dans la région historique de l'ancienne Samarie. Dans la Bible hébraïque, Bethel est lié notamment au passage des patriarches Abraham et Jacob sur ce lieu : en chemin vers Canaan, Abraham y construit un autel ; suite à son combat avec l'Ange, Jacob s'y endort et rêve de " l'échelle de Jacob », une échelle parcourue par des anges et qui s'élève jusqu'au ciel.

${ }^{31}$ Communication personnelle.

${ }^{32}$ Esther Stocker : née en 1974 en Italie, vit et travaille à Vienne (Autriche). A étudié à l'Académie des Beaux-Arts de Vienne et de Brera-Milan, puis au Art Center College of Design de Pasadena (Californie). Son travail est largement exposé en Europe, aux États-Unis et en Asie : Musée Vasarely (Budapest), musée Ritter (Waldenbuch), BF15 (Lyon), Setouchi Triennale (Japon), Kunsthalle (Bratislava), Nuit Blanche (Paris), musée Georg Kolbe (Berlin), etc. Esther Stocker est lauréate du Prix Aurélie Nemours 2020. Ce prix « récompense tout artiste, indépendamment de sa discipline, dont l'œuvre poursuit la quête plastique rigoureuse et empreinte de spiritualité qui fut celle d’Aurélie Nemours ». Du 23 janvier au 4 avril 2020, la quatrième exposition personnelle d'Esther Stocker, intitulée Loving imperfectly, a eu lieu dans l'espace parisien de la galerie Alberta Pane.
} 
L'œuvre Unlimited Space (fig. 6) est une installation qui investit la totalité d'une pièce à la structure improbable. Peinte sur le mur du fond, une grille faite de carrés blancs délimités par des traits noirs - à peu près régulière dans sa partie centrale, mais gagnée par le désordre sur ses bords. L'impression chaotique est d'autant plus forte que la grille se prolonge - partiellement et tant bien que mal - sur toutes les parois de la pièce, qui se fondent les unes dans les autres : en atteignant le sol et le plafond, ou les murs latéraux, les lignes perdent leur linéarité même. Les décrochages qui bousculent la structure orthogonale centrale sont, soit ponctuels et abrupts, soit hésitants, relevant davantage du gribouillage spontané que du geste maîtrisé. L'absence de limites claires entre des parois qui semblent s'avancer vers nous, la continuité des lignes en dépit de leurs accrocs, tout concourt à brouiller nos repères visuels, ce qui nous plonge effectivement dans un « espace illimité ».

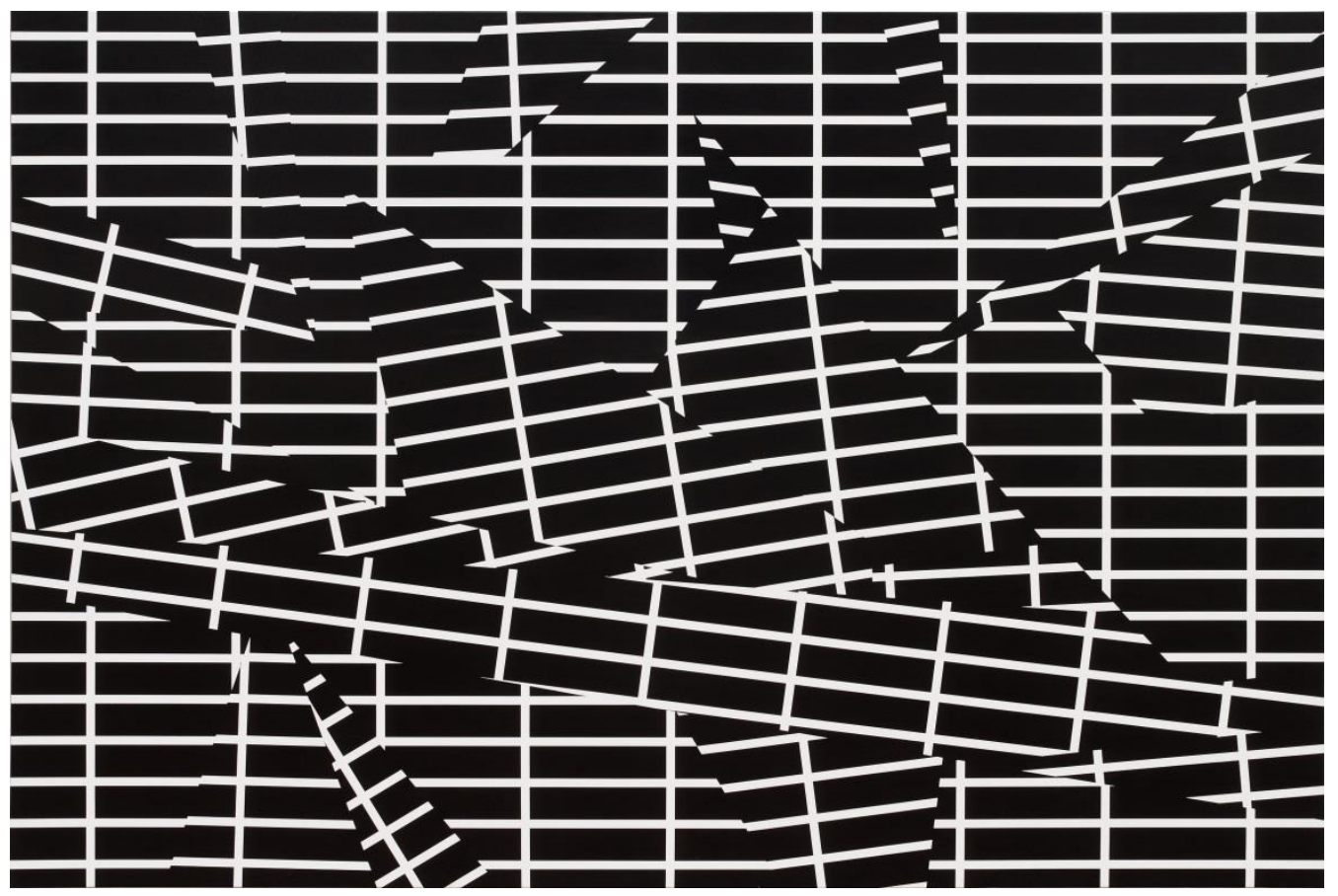

Figure 5. Esther Stocker, Sans titre, 2010, acrylique sur toile, $200 \times 300 \mathrm{~cm}$. Courtesy Galerie Alberta Pane.

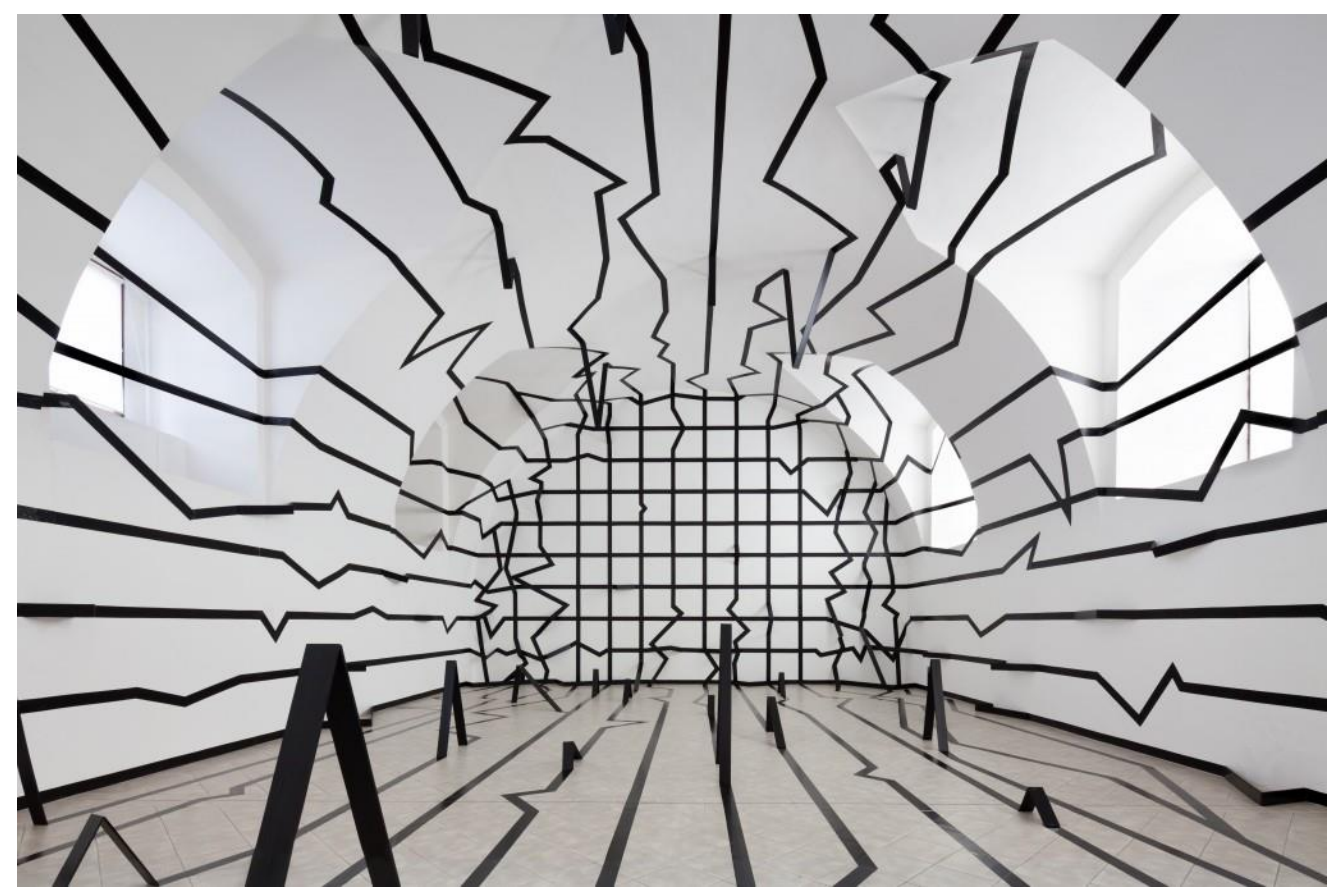

Figure 6. Esther Stocker, Unlimited Space, 2013, Roudnice, République tchèque. Courtesy Galerie Alberta Pane. 


\section{Au-delà de la géométrie}

Nous avons vu que la géométrie, à travers tous ses avatars, est la science des formes par excellence. Peut-on néanmoins envisager d'autres manières d'en rendre compte, qui la compléteraient ou la dépasseraient à certains égards, voire qui ne lui devraient rien ? La question se pose aux échelles extrêmes du monde physique, mais aussi en art.

Nous nous interrogerons sur la pertinence de l'abord géométrique pour les structures quantiques et cosmiques ; nous évoquerons la dialectique complexe des contours et des contenus formels ; nous toucherons enfin à l'essence indicible des formes, à travers quelques artistes qui semblent y avoir eu accès.

\section{Le quantique et le cosmique}

La mathématique représente l'origine par un point, qui est la forme géométrique à zéro dimension. Ce point géométrique s'applique également en physique nucléaire, mais non sans certaines difficultés, comme le souligne le physicien Gilles Cohen-Tannoudji : «l'expression "particules élémentaires" suggère des objets ponctuels, donc sans forme. C'est un des paradoxes de la physique de l'élémentarité que cette notion de particule sans forme s'est trouvée un peu contredite par les développements de la physique elle-même. [...] On s'est rendu compte que le noyau avait une certaine forme spatiale, une certaine extension, et que les constituants du noyau que sont les protons et les neutrons ont aussi une certaine extension spatiale, $[\ldots]$ une structure, qui s'est avérée granulaire. Le proton est fait de particules qui, apparemment, sont peut-être ponctuelles, et d'un niveau plus fondamental que le proton : ce sont les quarks. [...] D'une certaine façon, on peut dire que toute particule quantique a une certaine forme qui dépend de la résolution à laquelle on l'observe ${ }^{33}$. $\gg$ Au-delà du niveau quantique, à l'échelle supra-atomique, les molécules sont constituées d'atomes liés les uns aux autres pour former des polygones ou des polyèdres réguliers.

La forme originaire, qui est celle des constituants les plus fondamentaux de la matière, pourrait donc bien être ponctuelle. Qu'en est-il à l'échelle de l'univers ? Pour de nombreuses cosmogonies, la formation du monde équivaut strictement à l'apparition de la forme et à sa diversification à partir d'une origine unique, laquelle peut être considérée, soit comme informe - le tohu-bohu de la Genèse $^{34}$ (désordre, confusion de choses mêlées), le chaos de l'Antiquité grecque (Hésiode ${ }^{35}$, Ovide $^{36}$ ) ou l'eau chez Thalès ${ }^{37}$-, soit comme une forme simple, tel l'œuf cosmique ${ }^{38}$.

\footnotetext{
${ }^{33}$ Gilles Cohen-Tannoudji, « Formes et particules », dans Les sciences de la forme aujourd’hui, op. cit., p. 40-41, 43.

${ }^{34}$ Genèse 1,2 : « la terre était tohu-bohu / informe et vide, les ténèbres étaient au-dessus de l'abîme et le souffle de Dieu planait au-dessus des eaux. "

${ }^{35}$ Hésiode (VIII ${ }^{\text {ème }}$ s. av J.-C.) : « Donc, en premier naquit Chaos ; puis Gaïa aux larges flancs... », Théogonie, v. $116-117$.

${ }^{36}$ Ovide (-43 à 17) : « une masse grossière et confuse, rien d'autre qu'un amas inerte, un entassement de semences de choses, d'éléments divisés et mal joints ", Les Métamorphoses, 1,5.
}

${ }^{37}$ Thalès de Milet : philosophe et savant grec présocratique (-625 à -547). Considéré comme le premier philosophe de la nature, il est l'auteur de nombreuses recherches mathématiques, notamment en géométrie. Il considère que l'eau est le principe matériel explicatif de l'univers, d'où procèdent les autres éléments : feu, terre et air.

${ }^{38}$ CEuf cosmique : concept symbolique utilisé dans de nombreuses mythologies pour expliquer l'apparition du monde. Certains modèles cosmologiques actuels font l'hypothèse qu'il y a 13,7 milliards d'années la masse entière de l'univers était compressée en une singularité gravitationnelle, dite œuf cosmique (voir Jean Audouze, "Big bang et nucléosynthèse ", Revue Générale Nucléaire, $\mathrm{n}^{\circ} 4,1^{\mathrm{er}}$ août 2001, p. 14-17), qui s'est étendue vers son état actuel par le Big Bang. 
En termes modernes, on dira que lors de la structuration de 1'univers, c'est la transformation de l'énergie en matière qui a permis l'apparition des formes. De quoi dépendent donc les formes des objets cosmiques ? Essentiellement de la gravitation : les objets homogènes et massifs (étoiles et planètes, à partir d'un diamètre de 500 kilomètres) sont sculptés par la gravitation, qui leur confère une forme sphérique. Les corps plus petits (astéroïdes) ou très inhomogènes, composés par exemple de gaz et de poussières (noyaux de comètes), n'étant pas affectés par la gravitation, ont des formes non sphériques et souvent biscornues. Aux échelles encore plus grandes des amas de galaxies, selon l'astrophysicien Jean-Pierre Luminet, « la répartition à très grande échelle dans l'univers ressemble à de la "mousse de savon". En d'autres termes, il existerait d'immenses régions de l'espace à peu près sphériques, pratiquement dépourvues de matière - des sortes de bulles. Quant aux amas et superamas de galaxies, ils seraient confinés aux parois de ces bulles, ou aux intersections entre bulles adjacentes ${ }^{39}$.»

Que peut-on dire à l'heure actuelle sur la forme globale de l'univers ${ }^{40}$ ?

$\mathrm{Au} \mathrm{XIX}^{\text {ème }}$ siècle, les géométries non-euclidiennes, en proposant des modèles d'espaces courbes pour lesquels le $\mathrm{V}^{\text {ème }}$ postulat d'Euclide (sur les parallèles) ne s'applique pas, avaient déjà renouvelé le vieux débat sur la finitude ou l'infinitude de l'univers. Mais l'idée que l'univers est une entité géométrique qui a une forme, date du début du $\mathrm{XX}^{\mathrm{ème}}$ siècle, avec les théories de la Relativité. Celles-ci permettent en effet de penser un espace ou un univers d'extension finie, c'est-à-dire avec une circonférence, mais sans aucun bord - excepté la singularité temporelle souvent appelée Big Bang mais qui n'a jamais existé de manière stricte et qui demeure inaccessible. Si l'on prend toutefois au sérieux le modèle du Big Bang, selon lequel l'univers est en expansion à partir de cette singularité, il aurait la forme d'un entonnoir. Cependant le conditionnel s'impose car la seule géométrie de l'univers à laquelle l'astrophysique ait accès, est une géométrie locale (caractérisée par la courbure de l'espace considéré, non nécessairement identique en tout point). Or celle-ci ne renseigne pas sur la géométrie globale de l'univers, qui serait seule à même de dire s'il est fini ou infini, au moins dans une dimension... Par ailleurs la courbure spatiale invoquée par les astrophysiciens, concerne un espace idéal, totalement homogène ; or l'univers réel n'est pas identique au modèle idéal qui le représente - comme la Terre, il a ses creux et ses bosses. De plus, le fait que les sondages concernent toujours une zone limitée de l'espace, empêche d'accéder à la valeur moyenne de la courbure. Enfin, la notion même de moyenne dans un espace-temps à quatre dimensions tel que l'univers, est discutable.

\section{Contours et contenus}

En affirmant que l'univers peut être pensé comme une forme sans bord, la cosmologie relativiste exclut ipso facto de le penser comme un objet plongé dans l'espace. En effet, ce que décrit la forme d'un objet dans l'espace, c'est sa frontière externe - abstraction faite de sa taille, son emplacement, son orientation dans l'espace, ou d'autres propriétés telles que sa couleur et ses matériaux constitutifs. Avec la totalité formelle de l'univers, on touche donc aux limites de ce que peut actuellement la géométrie en termes de représentation et de calcul.

Une forme peut-elle exister pleinement en vertu de ses seuls contours, c'est-à-dire sans contenu matériel ou énergétique ? C'est le cas en géométrie, qui ne distingue pas entre formes vides et formes pleines; réciproquement, certaines matières ne prennent pas forme en raison de leur fluidité : les matières fluides n'ayant pas de frontières propres, seule l'imposition de limites

\footnotetext{
${ }^{39}$ Jean-Pierre Luminet, « Les formes cosmiques », dans Les sciences de la forme aujourd’hui, op. cit., p. 58

40 À partir des propos de Marc Lachièze-Rey et Jean-Philippe Uzan, émission « Forme de l'univers : le débat infini », La Méthode scientifique, France Culture, 14 janvier 2020.
} 
extérieures peut leur conférer une forme. Mais il peut arriver qu'un matériau produise sa propre forme - une possibilité qui a été exploitée par certains artistes. Ainsi le polyuréthane, si on lui ajoute $\mathrm{du}$ fréon, produit une mousse dont le volume augmente dans des proportions impressionnantes ; lorsque la réaction chimique est terminée, la mousse se fige et se solidifie en fonction du dosage, acquérant une forme qui échappe partiellement au sculpteur et dans laquelle se lit le mouvement expansif qui l'a produite. Cette découverte du sculpteur César (1965) est à l'origine des Expansions que l'on a pu voir au Centre Pompidou dans « César la rétrospective » (13 décembre 2017 - 26 mars 2018), parmi lesquelles l'Expansion 14 figure dans la collection du Centre Pompidou. Dans le même ordre d'idées, on citera les constructions de métal fondu ou coulé (1968-69) de Richard Serra $^{41}$, notamment ses montages de plomb, particulièrement instables et destinés tôt ou tard à s'affaisser.

En art, les formes ne se réduisent pas à leurs contours : leur stricte géométrie a toujours partie liée avec la matière et/ou la lumière. Comme le dit si justement Henri Focillon, «les matières comportent une certaine destinée ou, si l'on veut, une certaine vocation formelle. Elles ont une consistance, une couleur, un grain. Elles sont forme, comme nous l'indiquions, et, par là même, elles appellent, limitent ou développent la vie des formes de 1 ' $\mathrm{art}^{42}$. »

\section{De la forme au sublime}

Sur le chemin intérieur qui conduit de la forme perçue à l'idée du sublime, se trouve la simplicité - plus qu'un but à poursuivre, une évidence, qui s'est imposée notamment aux minimalistes. Le sculpteur Brancusi le reconnaît : «On arrive à la simplicité malgré soi, en s’approchant du sens réel des $\operatorname{choses}^{43}$. » Comme s'il fallait que l'artiste se déprenne partiellement de lui-même et se laisse envahir par la forme de son œuvre naissante.

En art comme en science, «s'approcher du sens réel des choses » peut aussi se faire en toute conscience : selon le biologiste et mathématicien D’Arcy Thompson, « du concept de forme, nous nous hissons vers la compréhension des forces qui lui ont donné naissance ${ }^{44}$. 》 Mais si cette remontée de la géométrie à la physique de la forme est bien une conquête de l'esprit sur l'opacité de la forme, elle ne saurait préparer à rencontrer le sublime en elle. Une telle rencontre peut être provoquée par l'expérience de la beauté, qui, pour le philosophe John Dewey émanerait de la forme, « comme une essence transcendante, pour en envelopper la matière ${ }^{45}$.

Elle peut aussi coïncider avec une prise de conscience d'ordre supérieur, comme chez Max Bill, dans cet extrait aussi précis que puissant, à lire en prenant son temps : «Les mystères de la problématique mathématique, l'ineffable de l'espace, l'éloignement ou la proximité de l'infini, la surprise d'un espace qui commence d'un côté et se termine par un autre, qui est en même temps le même, la limitation sans limites exactes, la multiplicité qui, malgré tout, forme une unité, l'uniformité qui s'altère par la présence d'un seul accent de force, le champ de forces composé de pures variables, les parallèles qui se coupent et l'infinité qui revient à elle-même comme présence,

\footnotetext{
${ }^{41}$ Richard Serra : né en 1939, vit et travaille à New York. Artiste minimaliste américain, connu surtout pour ses sculptures en métal. Appartient au groupe «Anti-Form » de la Côte Ouest. Lauréat de nombreuses distinctions à l'échelle internationale.

${ }^{42}$ Henri Focillon, op. cit., p. 51.

${ }^{43}$ Constantin Brancusi cité par Carola Gledion-Welcker, in Constantin Brancusi, Neuchâtel, éditions du Griffon, 1958, p. 219.

44 D’Arcy Thompson, Forme et croissance, trad. angl. Dominique Teyssié, édition établie et présentée par John Tyler Bonner, Paris, les éditions du Seuil, 2009, p. 268.

45 John Dewey, L'art comme expérience, Gallimard, « Folio Essais », trad. angl. Jean-Pierre Cometti et alii, 2010, p. 189-190. 
et encore le carré à nouveau avec toute sa solidité, la droite qui n'est troublée par aucune relativité, et la courbe qui en chacun de ses points forme une droite ; toutes ces réalités, qui en apparence n'ont rien à voir avec la vie quotidienne de l'homme, sont, malgré tout, d'une importance transcendantale. Ces forces que nous manions sont les forces fondamentales auxquelles tout ordre humain est soumis, et qui sont contenues précisément dans tout ordre connaissable.

La conséquence de toutes ces choses est un apport neuf au contenu de l'art actuel, car elles ne sont pas du pur formalisme, comme on le considère par erreur ; elles ne sont pas seulement des formes de beauté, mais pensée, idée, connaissance, converties en forme ; c'est-à-dire qu'elles ne sont pas des substances existant en surface, mais l'idée première de la structure du monde, du comportement en face de l'image que nous pouvons faire actuellement du monde ${ }^{46}$. »

Que l'on voie dans la forme une incarnation de la beauté ou « l'idée première de la structure du monde », c'est dans l'esprit humain tout entier qu'elle se déploie, bien au-delà de la géométrie. Kandinsky en a tiré la conséquence ultime : "La forme proprement dite, même si elle est parfaitement abstraite ou ressemble à une forme géométrique, a sa propre résonance intérieure. La forme est un être spirituel doué de propriétés qui s'y identifient. Un triangle (sans autres précisions : pointu, plat ou équilatéral) est un de ces êtres avec son parfum spirituel propre. Associé à d'autres formes, ce parfum se différencie, s'enrichit de nuances harmoniques mais reste au fond inchangé, comme le parfum de la rose que l'on ne saurait confondre avec celui de la violette. Il en est de même pour le cercle, le carré et toutes les autres formes possibles ${ }^{47}$. » Pour l'artiste sensible au sublime, la « résonance intérieure » des formes, est leur vie même.

\footnotetext{
${ }^{46}$ Max Bill, "La pensée mathématique dans l'art de notre temps », Pevsner, Vantongerloo, Bill, cat. exp., Zürich, Kunsthaus, 1949, trad. fr. in Max Bill, cat. exp. Paris, CNAC, 1969, p. 39-43.

${ }^{47}$ Vassily Kandinsky, Du spirituel dans l'art, et dans la peinture en particulier, 1909 ; chap. VI, « Le langage des formes et des couleurs », p. 116-117; rééd. Gallimard, Folio/Essais, 2005.
} 